\title{
Colour-magnitude diagrams of the post-core collapse globular clusters NGC 6256 and NGC 6717 (Palomar 9)
}

\author{
S. Ortolani ${ }^{1}$, B. Barbuy ${ }^{2}$, and E. Bica ${ }^{3}$ \\ 1 Università di Padova, Dept. di Astronomia, Vicolo dell'Osservatorio 5, I-35122 Padova, Italy \\ 2 Universidade de São Paulo, CP 3386, São Paulo 01060-970, Brazil \\ 3 Universidade Federal do Rio Grande do Sul, Dept. de Astronomia, CP 15051, Porto Alegre 91500-970, Brazil
}

Received June 10; accepted October 8, 1998

\begin{abstract}
We present photometry of the globular clusters NGC 6256 in $V, I$, NGC 6717, NGC 6256 in $V$, $I$, and NGC 6717 in $B, V$, obtaining cluster parameters. NGC 6256 has a post core-collapse structure, while such feature is suspected for NGC 6717 . Both clusters are projected on the bulge.

From the colour-magnitude diagrams for NGC 6256 we derive reliable cluster parameters which considerably revise its properties, with respect to previous literature. It has a blue-extended Horizontal Branch already observed in other post-core collapse clusters in the bulge, such as HP1. We derive a reddening of $E(B-V)=1.10$, a distance $d_{\odot} \approx 6.4 \mathrm{kpc}$, and an intermediate metallicity for NGC 6256 .

NGC 6717 presents a blue Horizontal Branch. We derive a reddening of $E(B-V)=0.23$ and a distance $d_{\odot} \approx 7.1 \mathrm{kpc}$, locating the cluster in the bulge. The photometry is deep and we study the luminosity function, which presents some evidence of depletion of Main Sequence stars. This effect is not unexpected for such a poorly populated Palomar cluster in the bulge.

We also estimate the cluster age from the magnitude difference between the horizontal branch and the turnoff. We obtain $\Delta V_{\mathrm{HB}}^{\mathrm{TO}}=3.7$, which would place this intermediate metallicity bulge globular as coeval with the halo.
\end{abstract}

Key words: Galaxy: globular clusters: NGC 6256; NGC 6717 (Palomar 9) — HR diagram

\section{Introduction}

NGC 6256 is also designated as GCL B1656-3702, BH208 (van den Bergh \& Hagen 1975) and ESO391-SC6. It is

\footnotetext{
* Observations collected at the European Southern Observatory - ESO, Chile.
}

sometimes referred to in the literature as Terzan 12, however we do not recommend this nomenclature: Terzan (1971), in a revision of his original lists of new bulge clusters (Terzan 1968, 1971a), assigned the name of Terzan 12 to this cluster, where the previously named Terzan 12 became Terzan 11, because Terzan 11 of the previous list was the same as Terzan 5. Terzan 12 studied in Ortolani et al. (1998) is instead at coordinates $\alpha_{1950}=18^{\mathrm{h}} 09^{\mathrm{m}} 14.0^{\mathrm{s}}$, $\delta_{1950}=-22^{\circ} 45^{\prime} 18^{\prime \prime}$, and Terzan 5 studied in Ortolani et al. (1996) has coordinates $\alpha_{1950}=17^{\mathrm{h}} 45^{\mathrm{m}} 00.3^{\mathrm{s}}, \delta_{1950}=$ $-24^{\circ} 47^{\prime} 46^{\prime \prime}$.

NGC 6256 is at $\alpha_{1950}=16^{\mathrm{h}} 56^{\mathrm{m}} 10.2^{\mathrm{s}}$, $\delta_{1950}=-37^{\circ} 02^{\prime} 49^{\prime \prime}$ and $l=347.792^{\circ}, b=3.306^{\circ}$.

The cluster structure is very concentrated, with $c=$ 2.50 , and it presents a post core-collapse morphology (Trager et al. 1995).

Alcaino (1983) presented a photographic $B$ vs. $(B-V)$ CMD of the cluster, where the horizontal branch (HB) level was close to the detection limit. He suggested that the cluster is metal-rich like 47 Tucanae. He derived $V_{\mathrm{HB}}=18.2$ and $E(B-V)=0.80$, and a distance of $11.0 \mathrm{kpc}$ from the Sun. Based on Alcaino's CMD, Webbink (1985) also presents $V_{\mathrm{HB}}=18.2$, but estimated a somewhat higher reddening of $E(B-V)=0.88$ which placed the cluster at $d_{\odot}=9.1$ from the Sun. Webbink lists a rather low metallicity for the cluster, $[M / H]=-1.56$. The compilation by Harris (1996) indicates $V_{\mathrm{HB}}=18.20$, $E(B-V)=0.84, d_{\odot}=8.9 \mathrm{kpc},[\mathrm{Fe} / \mathrm{H}]=-0.70$.

NGC 6717, also known as GCL B1852-2245, Palomar 9, GCl-105 (Alter et al. 1970), ESO523-SC14, has coordinates $\alpha_{1950}=18^{\mathrm{h}} 52^{\mathrm{m}} 05.2^{\mathrm{s}}, \delta_{1950}=-22^{\circ} 45^{\prime} 55^{\prime \prime}$ and $l=12.877^{\circ}, b=-10.900^{\circ}$.

The cluster structure is very concentrated, with $c=$ 2.07 , and it may have a post-core-collapse morphology (Trager et al. 1995).

Goranskii (1979) presented a photographic colourmagnitude diagram of NGC 6717 , where the horizontal 


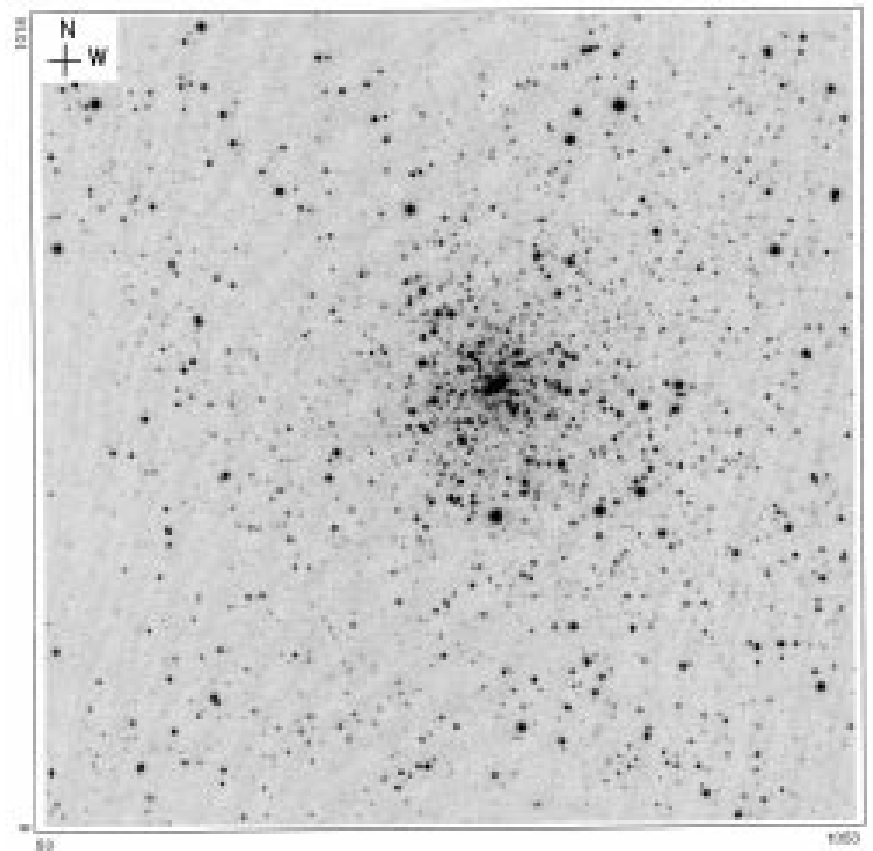

Fig. 1. $V$ image of NGC 6256. Dimensions are $6.3^{\prime} \times 6.3^{\prime}$. North is to the top and East is to the left

branch was close to the detection limit. He also studied a RR Lyrae star, concluding that it was a cluster member. A reddening of $E(B-V)=0.17$, a distance from the Sun of $d_{\odot}=7.6 \mathrm{kpc}$, and an estimated metallicity of $[M / H] \approx-1.3$ were derived. Based on Goranskii's CMD, Webbink (1985) indicated a $\mathrm{HB}$ level of $V_{\mathrm{HB}}=15.7$, which combined to a reddening of $E(B-V)=0.20$ from integrated colours, led to a distance from the Sun of $d_{\odot}=7.8 \mathrm{kpc}$. Webbink presents a lower metallicity of $[M / H]=-2.19$. Zinn \& West (1984) obtained an integrated spectrum of the cluster in the range 3600$5700 \AA$, estimating a reddening of $E(B-V)=0.22$, and a metallicity of $[\mathrm{Fe} / \mathrm{H}]=-1.32$. Harris's (1996) compilation gives $V_{\mathrm{HB}}=15.50, E(B-V)=0.21, d_{\odot}=6.6 \mathrm{kpc}$ and $[\mathrm{Fe} / \mathrm{H}]=-1.32$. Rutledge et al. (1997) derived a metallicity of $[\mathrm{Fe} / \mathrm{H}]=-1.32 \pm 0.15$, based on the near-infrared CaII triplet lines.

A deep CCD $B V$ photometry was presented by Brocato et al. (1996), showing that NGC 6717 has a moderately blue extended HB. They basically confirm the Zinn \& West (1984) values of reddening and metallicity.

In the present study we report $V, I$ photometry for NGC 6256 and $B, V$ photometry for NGC 6717 .

In Sect. 2 the observations are described. In Sects. 3 and 4 the clusters NGC 6256 and NGC 6717 are analysed respectively. The concluding remarks are provided in Sect. 5 .

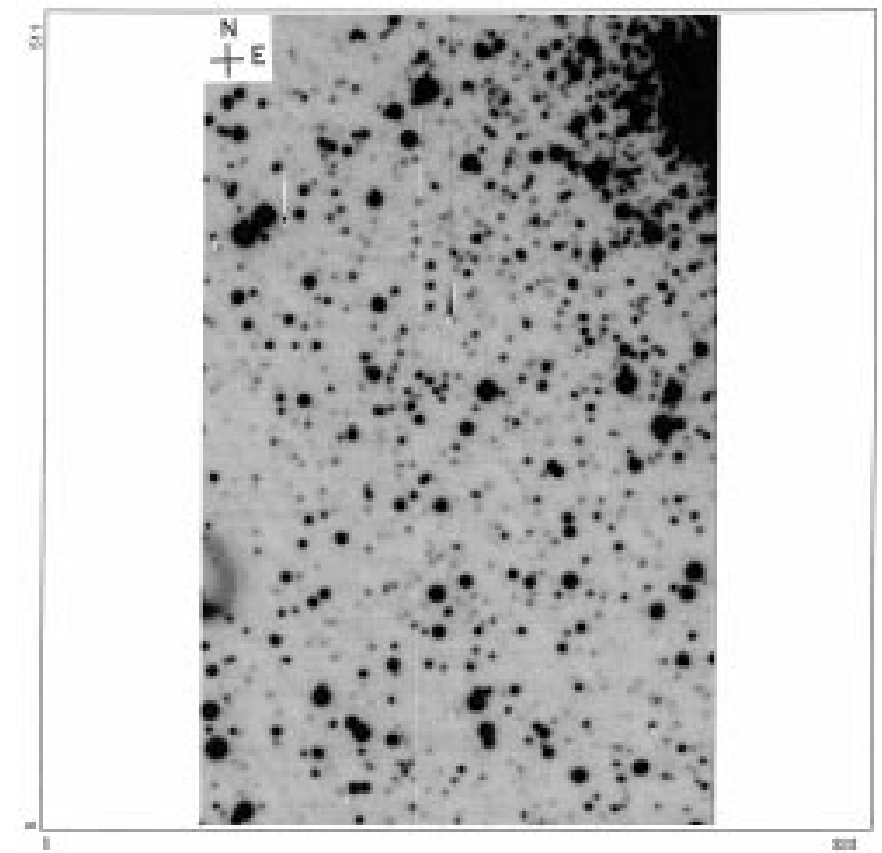

Fig. 2. $V$ image of NGC 6717. Dimensions are $4.0^{\prime} \times 2.5^{\prime}$. North is to the top and East is to the right

\section{Observations and reductions}

NGC 6256 and NGC 6717 were observed in 1994 May and 1985 June respectively with the $1.5 \mathrm{~m}$ Danish telescope at ESO (La Silla).

In the 1994 May run the Tektronix CCD ESO \# 28 of $1024 \times 1024$ pixels was used; the pixel size is $24 \mu \mathrm{m}$, corresponding to $0.37^{\prime \prime}$ on the sky, providing a full field of $6.3^{\prime} \times 6.3^{\prime}$.

In Fig. 1 is shown a $V 10$ min exposure of NGC 6256 . Notice the post core-collapse structure.

In the 1985 June run the $320 \times 512$ pixels RCA CCD ESO \# 1 was used. The pixel size is $30 \mu \mathrm{m}$, corresponding to $0.47^{\prime \prime}$ on the sky and the array gives a full field of $4^{\prime} \times 2.5^{\prime}$. In Fig. 2 the $V 15 \mathrm{~min}$ image of NGC 6717 is shown.

The log of observations is provided in Table 1.

Daophot II was used to extract the instrumental magnitudes. These magnitudes have been calibrated using Landolt (1983) stars for NGC 6717 and Landolt (1992) for NGC 6256 .

The reductions in the crowded reddened fields of NGC 6256 are described in detail in a study of Liller 1 (Ortolani et al. 1996 and references therein). The equations in the case of NGC 6256 are:

$V=26.91+0.04(V-I)+v$

$I=26.09+i$

reduced to $30 \mathrm{~s}$. exposure time and airmass of 1.1. Due to crowding effects arising in the transfer of the aperture magnitudes from standards to the field stars, the zero point calibration errors are dominant, estimated to 
Table 1. Log of observations

\begin{tabular}{llllll}
\hline Target & Filter & Date & Exp. & $\begin{array}{l}\text { Seeing } \\
(\mathrm{sec})\end{array}$ & $\left({ }^{\prime \prime}\right)$ \\
\hline NGC 6256 & $V$ & 19.05 .1994 & 30 & 1.1 \\
& $V$ & & 600 & 1.1 \\
& $B$ & & 60 & 1.2 \\
& $B$ & & 900 & 1.2 \\
& $I$ & & 20 & 1.1 \\
& $I$ & & 420 & 1.1 \\
NGC 6717 & $V$ & 16.06 .1985 & $2 \times 900$ & 1.9 \\
& $B$ & & $2 \times 1800$ & 1.9 \\
\hline
\end{tabular}

Note to the table: The long $B$ and $V$ observations of 24.06 .85 were taken offcenter, west of the cluster.

be about $\pm 0.03 \mathrm{mag}$ The CCD shutter time uncertainty $(0.7 \mathrm{~s})$ for a typical $20 \mathrm{~s}$ exposure time for the standard stars, produces an additional $3 \%$ uncertainty, which is propagated to the calibrations of the long exposure cluster frames. The final magnitude zero point uncertainty amounts to \pm 0.05 . The atmospheric extinction was corrected with the La Silla coefficients $\left(C_{V}=0.16\right.$, $C_{I}=0.12 \mathrm{mag} /$ airmass $)$. The zero point of the $V$ filter was checked relative to the Alcaino (1983) photometric stars and a good agreement within $0.02 \mathrm{mag}$ was found.

NGC 6717 was observed in the same run as Palomar 12 , and characteristics of the instruments and calibration procedures used are described in Gratton \& Ortolani (1988). The equations in the case of NGC 6717 are:

$B=24.88+0.16(B-V)+b$

$V=24.25+0.08(B-V)+v$

for exposures of $15 \mathrm{~s}$. in $V$ and $30 \mathrm{~s}$. in $B$ (airmass $=1.15)$. The atmospheric extinction was corrected with the standard La Silla coefficients $\left(C_{V}=0.14, C_{B}=\right.$ $0.22 \mathrm{mag} /$ airmass).

NGC 6717 has the bright field star $\nu_{2}$ Sgr $3^{\prime}$ to the north, which was placed outside the CCD frame; the cluster is offcenter but the star glare is avoided, and checks showed that in fact no diffuse light disturbed our frames.

\section{The globular cluster NGC 6256}

\subsection{Colour-magnitude diagrams}

Figures $3 \mathrm{a}$, b, c show a series of $V$ vs. $(V-I)$ CMD spatial extractions for the cluster.

Figure 3a corresponds to a ring with $9^{\prime \prime}<r<141^{\prime \prime}$. In the brighter features there is some field contamination but it is a deep photometry diagram where the turnoff (TO) is attained.

Figures $3 \mathrm{~b}$ and $3 \mathrm{c}$ correspond to extractions of $r<55^{\prime \prime}$ and $r<30^{\prime \prime}$ respectively. Notice the better definition of cluster sequences due to reduced field contamination. The cluster has a blue HB and there is no evident red HB. The
Table 2. Counts of Blue HB, SGB and field MS stars in frames of different aperture

\begin{tabular}{lllll}
\hline aperture & BHB & SGB & HB/SGB & diskMS \\
\hline$R>150$ & 30 & 162 & 0.18 & 40 \\
$R>100$ & 43 & 182 & 0.23 & 41 \\
$R<100$ & 19 & 22 & 0.86 & 2 \\
$R<80$ & 12 & 19 & 0.63 & 0 \\
\hline
\end{tabular}

presence of a blue HB is made more clear in Fig. 3c where essentially no blue MS contamination is seen, and stars in the locus of the blue HB are still seen.

A quantitative check of the presence of an HB was carried out by counting stars in frames with different apertures around the cluster center. In Table 2 are given the numbers corresponding to: $\mathrm{HB}$ contained in the box of stars bluer than $V-I<1.6$ and in the magnitude interval $18.2<V<19.8$ and for the field the stars in a box defined along the subgiant branch (SGB) at the same magnitude level, and in the colour interval $2.8<(V-I)<3.3$; in order to check if there is any serious selection due to colour counts were made of the blue MS disk field stars, with a selection of $V<18.2$ and $(V-I)<1.6$ (same colour as BHB). The numbers are self explaining. There is a big jump from the $R>150$ pixels (which is almost pure field) and $R<100$. The slow decline at $R<80$ could be just a stochastic fluctuation and/or a selection effect (loosing blue stars because of crowding in $V$ ). The strong relative concentration of the $\mathrm{BHB}$ stars relatively to the disk stars is evident as well, the ratio BHB/SGB above is not affected by colour selection.

The blue HB appears to be extended, showing a similar morphology to intermediate metallicity clusters in other bulge clusters like NGC 6522 (Barbuy et al. 1994; Terndrup \& Walker 1994; Shara et al. 1998) and HP 1 (Ortolani et al. 1997). Notice that the RGB of NGC 6256 is also sparsely populated.

In Fig. $3 \mathrm{~b}$ is superimposed the mean locus of the post core-collapse globular cluster NGC 6752 from Rosino et al. (1997) of $[\mathrm{Fe} / \mathrm{H}]=-1.54($ Zinn \& West 1984). A good agreement is obtained for the cluster sequences.

Recently, Bica et al. (1998) carried out a near-infrared integrated spectral study of this cluster, having obtained $[\mathrm{Fe} / \mathrm{H}]=-1.01$. The cluster appears to be more metalpoor than 47 Tuc.

We adopt a compromise value for the cluster metallicity of $[\mathrm{Fe} / \mathrm{H}] \approx-1.3$.

\subsection{Reddening and distance}

The brighter parts of the blue HB of NGC 6256 are located at $V_{\mathrm{HB}}=18.0 \pm 0.15$.

We calculate the cluster reddening taking NGC 6752 as reference. By matching the overall sequences (Fig. 3b) 

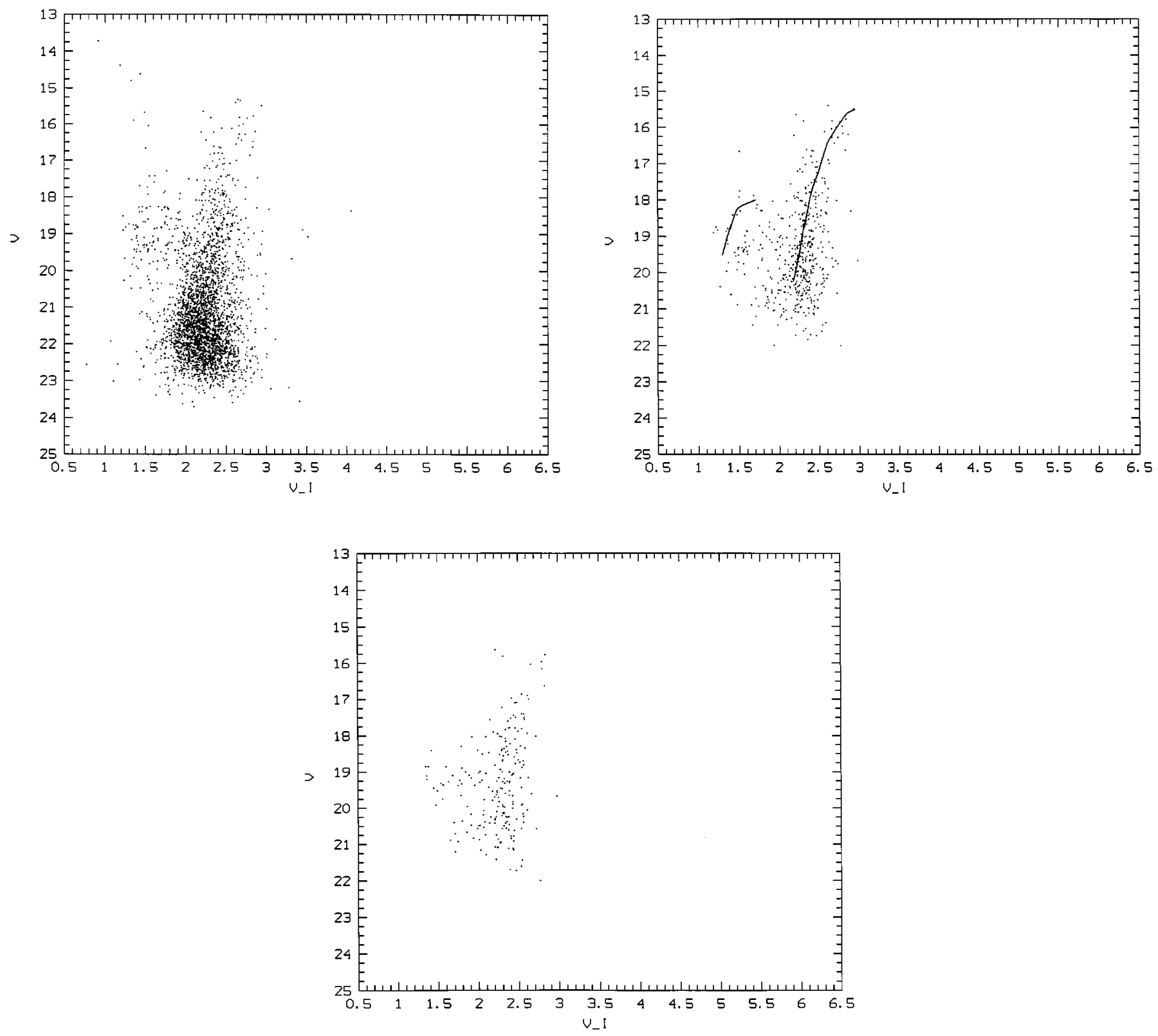

Fig. 3. $V$ vs. $(V-I)$ CMD for NGC 6256: a) $9^{\prime \prime}<r<141^{\prime \prime}$ b) extractions of $r<55^{\prime \prime}$, together with mean locus of NGC 6752 overplotted, c) extraction of $r<30^{\prime \prime}$

we obtain $\Delta(V-I)=1.40$. Adopting $E(V-I) / E(B-$ $V)=1.31$ (Dean et al. 1978), this corresponds to $\Delta(B-$ $V)=1.07$, and using $E(B-V)=0.03$ for NGC 6752 (Harris 1996), we get $E(B-V)=1.10$, which corresponds to $A_{V}=3.41\left(R=A_{V} / E(B-V)=3.1\right.$ cf. Savage \& Mathis 1979). This value is somewhat higher than Alcaino's (1983) value of $E(B-V)=0.80$, which was caused by the use of the 47 Tuc RGB as reference. Recently Bica et al. (1998) carried out an integrated spectroscopic study of reddened bulge clusters in the near-IR. For NGC 6256 they obtained $E(B-V)=0.95$; this value is somewhat lower than the CMD value derived here, possibly due to crowding and/or contamination effects.
Adopting an absolute magnitude of the $\mathrm{HB}$ for NGC 6752 of $M_{V}=0.7$ (Buonanno et al. 1989, we get the absolute distance modulus $(m-M)_{0}=13.89 \pm 0.2$. This corresponds to a distance of $d_{\odot}=6.0 \pm 0.6 \mathrm{kpc}$ for NGC 6256. A slightly larger distance is obtained if the absolute distance modulus of NGC 6752 determined by Renzini et al. (1996) by means of white dwarfs is used $\left(d_{\odot}=6.7 \mathrm{kpc}\right)$. We adopt a compromise value of $d_{\odot}=6.4 \pm 0.5 \mathrm{kpc}$ for NGC 6256. The Galactocentric coordinates of the cluster, assuming a distance of the Sun to the Galactic center of $R_{\odot}=8.0 \mathrm{kpc}$ (Reid 1993), are $X=-1.8$ ( $X<0$ refers to our side of the Galaxy), $Y=-1.3 \mathrm{kpc}$ and $Z=0.4 \mathrm{kpc}$. NGC 6256 is thus 


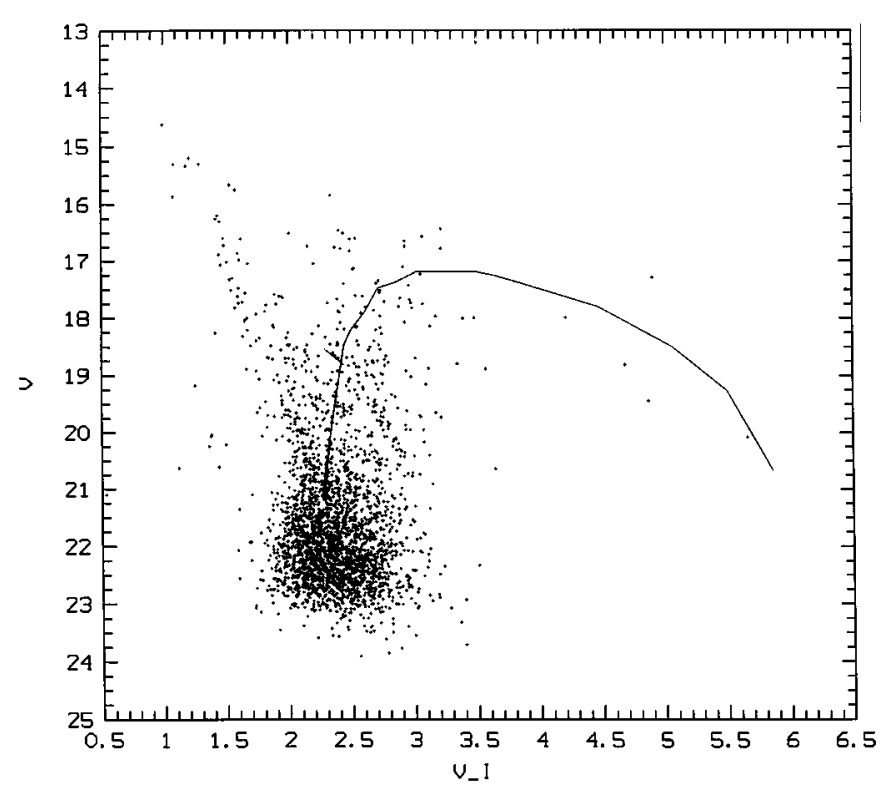

Fig. 4. Surrounding field population $\left(r>188^{\prime \prime}\right)$. The mean locus of the bulge cluster NGC 6553 is overplotted

located in the bulge, but does not belong to the metal-rich population.

\subsection{The surrounding field}

The field surrounding NGC 6256 is interesting because it is near the galactic plane $\left(b \approx 3.3^{\circ}\right)$ along the bulge major axis $\left(l=-12 \cdot 2^{\circ}\right)$.

We show in Fig. 4 an extraction for $r>188^{\prime \prime}$, basically corresponding to the field population. We see a bulge TO, and a cool extended RGB for $(V-I)>3.5$.

The field population can be matched with the mean locus of the nearly solar metallicity globular cluster NGC 6553, which in turn is similar to that of Baade's Window (Ortolani et al. 1995). Therefore this piece of information suggests for the present field located $\approx 12^{\circ}$ from the Galactic center along the major axis, a comparable metallicity to that of Baade's Window (which is located at $\approx 4^{\circ}$ from the Galactic center along the minor axis).

From the fit to NGC 6553 (Fig. 4) we can estimate a reddening difference of $\Delta(V-I)=0.5 \pm 0.2$, and adopting $E(B-V)=0.7$ for NGC 6553 (Guarnieri et al. 1998) we get $E(B-V)=1.1 \pm 0.2$ for the present field (thus comparable to that of NGC 6256 itself). Since we assumed that the field is as metal-rich as NGC 6553, there might be an additional error of $\sim 0.2$ in $E(B-V)$ if the metallicity of the field were as low as that of NGC 6752 , due to a blanketing difference relative to NGC 6553; however such low metallicity for the bulge is unlikely.

This adds a new point in the reddening mapping of different directions in the bulge, as deduced from CMDs of clusters and fields (Barbuy et al. 1998 and references therein).

Finally, we note that a peculiarity of this field is the lack of a defined HB, probably due to low statistics and differential reddening effects.

\section{The globular cluster NGC 6717 (Palomar 9)}

\subsection{Colour-magnitude diagrams}

Brocato et al. (1996) studied the brighter CMD sequences of this cluster in detail, while the aim of the present study is to explore the $\mathrm{HB}$ and lower sequences.

Figures $5 \mathrm{a}$, b show $V$ vs. $(B-V)$ CMD spatial extractions for the cluster, corresponding to the whole frame $\left(4.0^{\prime} \times 2.5^{\prime}\right)$, and to a ring with $19^{\prime \prime}<r<94^{\prime \prime}$ (in Fig. $5 \mathrm{~b}$ the cluster crowded core is avoided), respectively. In Fig. 5a the field contamination is rather important while in Fig. 5b the cluster sequences are well defined. The mean locus of NGC 6752 from Desidera et al. (1997) is superimposed on the diagram in Fig. 5b.

Again, like in the case of NGC 6256, we use NGC 6752 as reference. In Fig. 5b is superimposed the mean locus of the post core-collapse globular cluster NGC 6752 from Buonanno et al. (1986). A good agreement is obtained for the cluster sequences. Bica et al. (1998) spectroscopy gives $[\mathrm{Fe} / \mathrm{H}]=-1.05$. Likewise for NGC 6256 , we adopt a compromise value for the cluster metallicity of $[\mathrm{Fe} / \mathrm{H}] \approx-1.3$ for NGC 6717.

\subsection{Reddening and distance}

The brighter parts of the blue HB of NGC 6717 are located at $V=15.55 \pm 0.15$.

By matching the fiducial sequence of NGC 6752 (Buonanno et al. 1986; Desidera et al. 1997) to the cluster CMD (Fig. 5b) we obtain $\Delta(B-V)=0.20$. With $E(B-V)=0.03$ for NGC 6752 there results $E(B-V)=0.23$, which corresponds to $A_{V}=0.71$. Bica et al. (1998) using the continuum distribution of a near-IR integrated spectrum gives $E(B-V)=0.21$. The results are comparable within uncertainties.

Adopting an absolute magnitude of the $\mathrm{HB}$ for NGC 6752 of $M_{V}=0.7$ (Buonanno et al. 1989), we derive an absolute distance modulus $(m-M)_{0}=14.14 \pm 0.2$. This corresponds to a distance of $d_{\odot}=6.7 \pm 0.6 \mathrm{kpc}$ for NGC 6717. Assuming an absolute distance modulus of NGC 6752 as determined by Renzini et al. (1996) we obtain a distance of $d_{\odot}=7.5 \mathrm{kpc}$ for the present cluster. We adopt a compromise value of $d_{\odot}=7.1 \pm 0.5 \mathrm{kpc}$ for NGC 6717. The Galactocentric coordinates are $X=-1.2$, $Y=1.5 \mathrm{kpc}$ and $Z=-1.3 \mathrm{kpc}$. Therefore NGC 6717 is located in the bulge. 

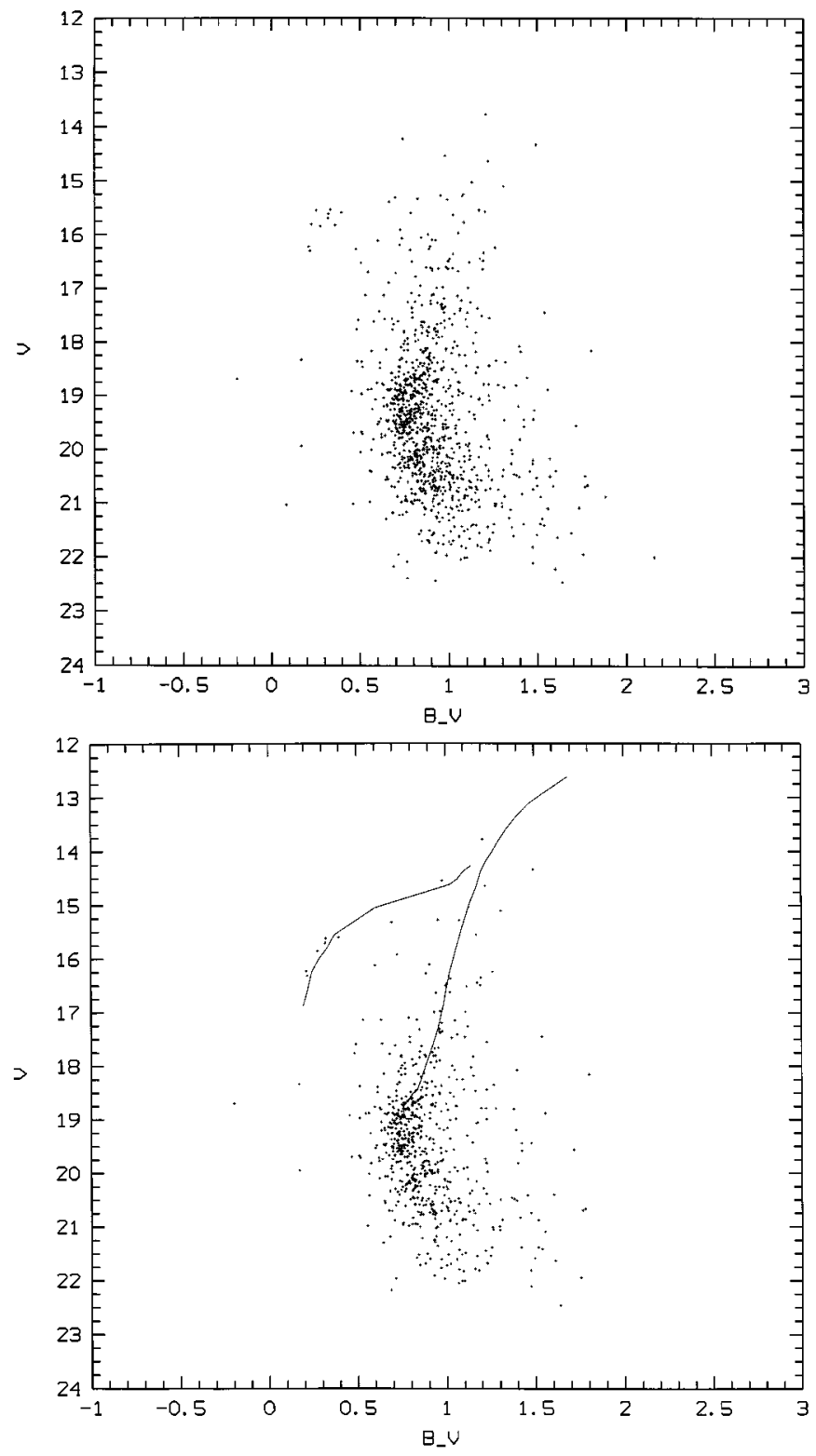

Fig. 5. NGC 6717: a) whole frame; b) extraction of $19^{\prime \prime}<r<$ $94^{\prime \prime}$, with mean locus of NGC 6752 overplotted

\subsection{Age}

The magnitude difference between the horizontal branch and the turnoff has been pointed out as a good age indicator (Iben 1974; Sandage 1986 and references therein).

For NGC 6717 we obtain from Fig. $5 \mathrm{~b} \Delta V_{\mathrm{HB}}^{\mathrm{TO}} \approx$ $3.7 \pm 0.2$, comparable to the mean value for the halo of $\Delta V_{\mathrm{HB}}^{\mathrm{TO}} \approx 3.55$ by Buonanno et al. (1989). This indicates that NGC 6717 is coeval with the halo, within uncertainties.

Adopting the present reddening, distance modulus, and apparent total $V$ magnitude $V_{\mathrm{t}}=9.28$ (Harris 1996), we derive an absolute integrated magnitude for this cluster $M_{V}=-5.57$. NGC 6717 is not an extremely low mass globular cluster like E3 (McClure et al. 1985; Gratton \& Ortolani 1987), but it is comparable to halo Palomar clusters. This is a rare case of such type of clusters in the bulge.

\subsection{Variable stars}

Goranskii (1979) discovered an RR Lyrae star in this cluster, and constructed a light curve. The RR Lyrae is outside our frame. Placing his data for this star of $V=15.7$ and $(B-V)=0.5$ on our CMD, we see that it is at the correct magnitude level of the HB, and at the RR Lyrae gap, and can be considered a member. Goranskii determined a period of 0.5752 days, so that the cluster can be tentatively classified as Oosterhoff type I.

\section{Conclusions}

Cluster parameters based on CCD photometry were derived for NGC 6256 and NGC 6717 . They are both located in the bulge, but are not metal-rich.

A new family of clusters in the bulge appears to be revealed from a series of recent studies. They show blue extented HBs, post core-collapse structure confirmed or suspected and intermediate metallicities. With the present study the two clusters NGC 6256 and NGC 6717 can be added to this class.

The other members showing these characteristics are HP 1 (Ortolani et al. 1997), NGC 6522 (Barbuy et al. 1994; Terndrup \& Walker 1994), NGC 6540 (Bica et al. 1994) and NGC 6558 (Rich et al. 1998).

The identification of this family in the bulge was not recognized in early CMDs because they did not attain the blue extended HB.

Acknowledgements. BB and EB acknowledge partial financial support from $\mathrm{CNPq}$ and Fapesp.

\section{References}

Alcaino G., 1983, A\&AS 53, 47

Alter G., Ruprecht J., Vanysek V., 1970, Catalogue of Star Clusters and Associations, Alter G., Balasz B., Ruprecht J. (eds.). Akademiai Kiado, Budapest

Barbuy B., Ortolani S., Bica E., 1994, A\&A 285, 871

Barbuy B., Bica E., Ortolani S., 1998, A\&A 333, 17

Bica E., Clariá J.J., Piatti A.E., Bonatto C., 1998, A\&AS 131, 483

Bica E., Ortolani S., Barbuy B., 1994, A\&A 283, 67

Brocato E., Buonanno R., Malakhova Y., Piersimoni A.M., 1996, A\&A 311, 778

Buonanno R., Caloi V., Castellani V., Corsi C., Iannicola G., Fusi Pecci F., Liller W, 1986, A\&AS 66, 79

Buonanno R., Corsi C.E., Fusi Pecci F., 1989, A\&A 216, 80

Dean J.F., Warren P.R., Cousins A.J., 1978, MNRAS 183, 569 
Desidera S., Bertelli G., Ortolani S., 1997, in IAU Symp. 189, Fundamental Stellar Parameters: confrontation between theory and observations, Bedding T., Booth A., Davis J. (eds.), poster papers, p. 164

Goranskii V.P., 1979, Soviet Astr. 23, 284

Gratton R.G., Ortolani S., 1987, A\&AS 67, 373

Gratton R.G., Ortolani S., 1988, A\&AS 73, 137

Guarnieri M.D., Ortolani S., Montegriffo P., Renzini A., Barbuy B., Bica E., Moneti A., 1998, A\&A 331, 70

Harris W.E., 1996, AJ 112, 1487

Iben I.Jr., 1974, ARA\&A 12, 215

Kunkel W.E., 1980, ApJ 239, 112

Landolt A.U., 1983, AJ 88, 439

Landolt A.U., 1992, AJ 104, 340

McClure R.D., Hesser J.E., Stetson P.B., Stryker L.L., 1985, PASP 97, 665

Ortolani S., Bica E., Barbuy B., 1996, A\&A 306, 134

Ortolani S., Bica E., Barbuy B., 1997, MNRAS 284, 692

Ortolani S., Bica E., Barbuy B., 1998, A\&AS 127, 471

Ortolani S., Renzini A., Gilmozzi R., Marconi G., Barbuy B., Bica E., Rich R.M., 1995, Nat 377, 701

Peterson C.J., 1986, PASP 98, 1258
Renzini A., Bragaglia A., Ferraro F.R., Gilmozzi R., Ortolani S., Holmberg J.B., Liebert J., Wesemael F, Bohlin R.C., 1996, ApJ 465, 23

Reid M., 1993, ARA\&A 31, 345

Rich R.M., Ortolani S., Bica E., Barbuy B., 1998, AJ 116, 1295

Rosino L., Ortolani S., Barbuy B., Bica E., 1997, MNRAS 289, 745

Rutledge G.A., Hesser J.E., Stetson P.B., 1997, PASP 109, 907

Sandage A., 1986, ARA\&A 31, 345

Shara M., Drissen L., Rich R.M., Paresce F., King I.R., Meylan G., 1998, ApJ 495, 796

Savage B., Mathis J., 1979, ARA\&A 17, 73

Terndrup D.M., Walker A.R., 1994, AJ 107, 1786

Terzan A., 1968, Comptes Rendus Acad. Sci. Paris 267, 1245

Terzan A., 1971, A\&A 12, 457

Terzan A., 1971, A\&A 15, 336

Trager S.C., King I.R., Djorgovski S, 1995, AJ 109, 218

Webbink R.F., 1985, in Dynamics of Star Clusters IAU Symp. 113, Goodman J., Hut P. (eds.). Dordrecht: Reidel, p. 541 van den Bergh S., Hagen G.L., 1975, AJ 80, 11

Zinn R., West M.J., 1984, ApJS 55, 45 